\title{
Clinical Utility of Erythrocyte Sedimentation Rate Test
}

\author{
Tomomi Tabuchi ${ }^{4}$; Noriyuki Tatsumi ${ }^{1}$; Hiroshi Tominaga ${ }^{1}$; Akira Furota ${ }^{1}$; Norifumi Iwata ${ }^{2}$; and Genzi Suganuma ${ }^{3}$ \\ ${ }^{1}$ Department of Laboratory Medicine, Osaka City University Medical School, ${ }^{2}$ Midori Health Control Center, Osaka, ${ }^{3}$ Souken Co. (NPO), Tokyo, \\ and ${ }^{4}$ Nagase Co. Ltd., Osaka
}

\begin{abstract}
The Erythrocyte sedimentation test is a common method used worldwide to detect acute inflammation. ICSH-WHO reference and standard methods have been routinely used for many years. However, they require much blood and long testing time over 2 hours, and several simple and rapid methods for what have recently been proposed. This paper describes advantages and disadvantages of these methods, plasma factors affecting ESR and clinical utility of which test.
\end{abstract}

Key Words ESR; ICSH-WHO Method; Rapid Test; Inflammation Marker

\section{INTRODUCTION}

The erythrocyte sedimentation rate test (ESR) is one of the most common and traditional laboratory tests in the world. The ESR reflects both plasma concentration of acute-phase proteins of large molecular size and anemia. ${ }^{1)}$ The increased mobility of patients and the benefits to laboratories of sharing their experience have led to the need for comparability of measurements between laboratories. Thus, an original ICSH (International Council for Standardization in Hematology) reference method for measuring ESR based on the Fahraeus and Westergren method using diluted blood (4 vol. of blood plus 1 vol. of sodium citrate) in open-ended glass tubing of $300 \mathrm{~mm}$ in length, mounted vertically in a rack or stand was developed. ${ }^{2)}$ Modifications, in particular the use of undiluted blood, were recommended as the basis of a new ICSH reference standard. Responding to this recommendation, the ICSH reference method was released in 1988, and is performed with undiluted blood samples with hematocrit of 0.35 or less under standardized conditions in a Westergren open-ended glass pipette that meets ICSH specifications. These undiluted blood samples are anti-coagulated with EDTA (dilution less than $1 \%$ ). ${ }^{3)}$ For comparison with the traditional method using diluted blood, a correlation formula can be applied: diluted blood ESR $\mathrm{mm}=$ (undiluted blood ESR $\mathrm{mm} \times 0.86$ ) -12 . On the other hand, a new standardized method was proposed by the ICSH, which is designed for use of EDTA-ant coagulated and undiluted blood in pipettes with a $200 \mathrm{~mm}$ scale and to avoid spillage of blood or aerosol generation. ${ }^{1)}$ In 1993, the ICSH recommended specifications for working methods, using diluted or undiluted blood, which may be considered ICSH selected methods for routine use. ${ }^{1,4)}$ A protocol is outlined for evaluation of such working methods against the ICSH reference method or the new ICSH standardized method. ${ }^{5)}$

Received: May 11, 2001

Address; Nagase Co. Ltd., 1-17, Shinmachi, 1-Chome, Nishi-ku, Osaka, 550-8668 Japan. TEL; 81-6-6535-2612, FAX; 81-6-6535-2470
In contrast to the international movement for ESR testing, Asian countries including Japan have thus far been supporting the use of the traditional Westergren method ${ }^{6}$ for diluted blood as their routine method. The Westergren method is popular in Asian countries because it is simple, reliable, and inexpensive in the routine laboratory. When we searched the literature on ESR in the past 10 years through the PubMed site, over 500 were found to have been released every year. This reflects the fact that the ESR test still has clinical usefulness as a diagnostic tool.

\section{MERITS AND DEMERITS OF CONVENTIONAL WESTERGREN ESR TEST AND RAPID ESR TESTS}

In general, the Westergren method is known to detect inflammation markers. It required $2 \mathrm{~m} l$ diluted sample blood, a standardized Westergren tube, and at least 2-hour testing time. The tube must be stood strait-up and vertically at room temperature. Excessive sample number and time requirement are disadvantages for routine use. Spilling of blood is bio-hazardous for medical technologists. Furthermore, some researchers believe that ESR is less informative and cannot be automated, and that C-reactive protein determination is more useful that ESR tests for detection of acute inflammation, ${ }^{7)}$ although ESR testing is legally required for diagnosing and monitoring tuberculosis in Japan.

To compensate for such disadvantages, several types of simplified ESR methods have been proposed (Table 1). The first one developed was the Zeta Sedimentation Ratio (Zeta-fuge), by Dr. Bull. ${ }^{8-10)}$ Anti-coagulated blood $(0.18 \mathrm{ml})$ is inserted into an open-ended tiny glass tube $(75 \mathrm{~mm} \times 2.3 \mathrm{~mm})$, and the tube is loaded onto a specific centrifuge and centrifuged for 3 min. at room temperature. The results obtained are influenced by temperature, and not by hematocrit, but it is very difficult to determine the sedimentation ratio reading particularly for specimens judged normal by the Westergren method, because such specimens exhibit very small amounts of sedimentation. Furthermore, Zeta-fuge results exhibit a non-linear correlation with Westergren values. ${ }^{10)}$

The Ves-matic 20/60 (DIESSE Diagnostics Senese; SienaItaly) is an automatic instrument for the determination of ESR for 20 or 60 samples. One $\mathrm{m} l$ citrated blood is placed into a specific tube. Its tube loader holds sample tubes at a tilted angle and moves them automatically. The ESR reading is performed automatically, and testing time is less than $30 \mathrm{~min}$. for the Westergren 1-hour value and $40 \mathrm{~min}$. for the Westergren 2hour value. The results obtained with automated reading can be corrected by test temperature by means of simple keyboard 
Table 1 Inter-methodological comparison of ESR tests.

\begin{tabular}{|l|l|l|l|l|l|l|}
\hline & Westergren & Zeta-fuge & Ves-matic & Test-1 & Quick-Eye & HOK405 \\
\hline Sample & citrated/ & EDTA & citrated & EDTA & $\begin{array}{l}\text { citrated/ } \\
\text { EDTA }\end{array}$ & citrated \\
\hline Blood volume & $2 \mathrm{~m} l$ & $0.18 \mathrm{~m} l$ & $1 \mathrm{~m} l$ & $0.15 \mathrm{~m} l$ & $1.4 \mathrm{~m} l$ & $2 \mathrm{~m} l$ \\
\hline Test time & $60,120 \mathrm{~min}$. & $3 \mathrm{~min}$. & $20,40 \mathrm{~min}$. & $32 \mathrm{sec}$. & $20,40 \mathrm{~min}$. & $6,9 \mathrm{~min}$. \\
\hline $\begin{array}{l}\text { Automatic } \\
\text { processing }\end{array}$ & no & no & yes & yes & yes & no \\
\hline $\begin{array}{l}\text { Technical } \\
\text { simplicity }\end{array}$ & $\begin{array}{l}\text { relatively } \\
\text { simple }\end{array}$ & $\begin{array}{l}\text { relatively } \\
\text { simple }\end{array}$ & simple & simple & simple & $\begin{array}{l}\text { relatively } \\
\text { simple }\end{array}$ \\
\hline $\begin{array}{l}\text { Correlation } \\
\text { with } \\
\text { Westergren } \\
\text { method }\end{array}$ & 1.0000 & 0.9487 & $0.9680^{*}$ & 0.9538 & 0.9828 & 0.9112 \\
\hline $\begin{array}{l}\text { Laboratory } \\
\text { safety }\end{array}$ & no & no & yes & yes & yes & no \\
\hline $\begin{array}{l}\text { Graphic } \\
\text { analysis }\end{array}$ & no & no & no & no & yes & no \\
\hline $\begin{array}{l}\text { Temperature } \\
\text { control }\end{array}$ & needed & needed & not needed & not needed & not needed & needed \\
\hline $\begin{array}{l}\text { Others } \\
\text { and incorrect } \\
\text { reading } \\
\text { procedures }\end{array}$ & $\begin{array}{l}\text { in } \\
\text { meniscus } \\
\text { reading }\end{array}$ & $\begin{array}{l}\text { throughput } \\
\text { low reliability }\end{array}$ & throughput \\
$110 / \mathrm{h}$ & throughput \\
$30 / \mathrm{h}$ & $\begin{array}{l}\text { difficulty } \\
\text { in } \\
\text { meniscus } \\
\text { reading }\end{array}$ \\
\hline
\end{tabular}

S. Motiduki QC in ESR Proc. $41^{\text {st }}$ JPN. Soc. Clin. Pathol. 1994

operation. $^{11)}$

The Test 1 automated system was developed by Plebani's group (SIRE Analytical System, Uden-Italy). ${ }^{12)}$ EDTA blood $(150 \mu l)$ is first rotated 2 minutes, aspirated through an automated piecing system, distributed in a capillary and then centrifuged at about $20 \mathrm{~g}$. The system uses an infrared ray micrometer with a light wavelength of $950 \mathrm{~nm}$. Its sensing area temperature is maintained at $37^{\circ} \mathrm{C}$. Electrical impulses collected using a photodiode detector are directly correlated to the concentrations of RBCs present at capillary level. Its analytic throughput is a rate of 110 specimens per hour in continuous loading, providing a finding every 32 seconds. In our evaluation, the values of TEST 1 were lower than those obtained by the Westergren method.

The Quick-Eye was developed by Nagase Co. (TokyoJapan). ${ }^{13), 14)}$ EDTA or citrated blood is placed into a specific short glass tube, and tubes are loaded on a tilted holder. The holder is moved automatically, and red cell sedimentation is monitored every $40 \mathrm{sec}$. using a photodiode light detector. The Westergren 1-hour value is measured at $20 \mathrm{~min}$. and the Westergren 2-hour time is measured at $40 \mathrm{~min}$. after sample loading. The values correlated well with Westergren values. The meniscus between RBCs and plasma is separated clearly enough that its reading is very reliable. One characteristic of the Quick-Eye is that it is able to draw a graphic curve of the blood sedimentation process (Fig. 1). The graph is informative for observers and researchers in determining the sample specificity of sedimentation.

The HOK405 (Drager \& Heerhorst GmbH \& Co. KG) uses the same principle as the Westergren method. A Westergren glass tube filled with citrated blood is set on a tube holder, and
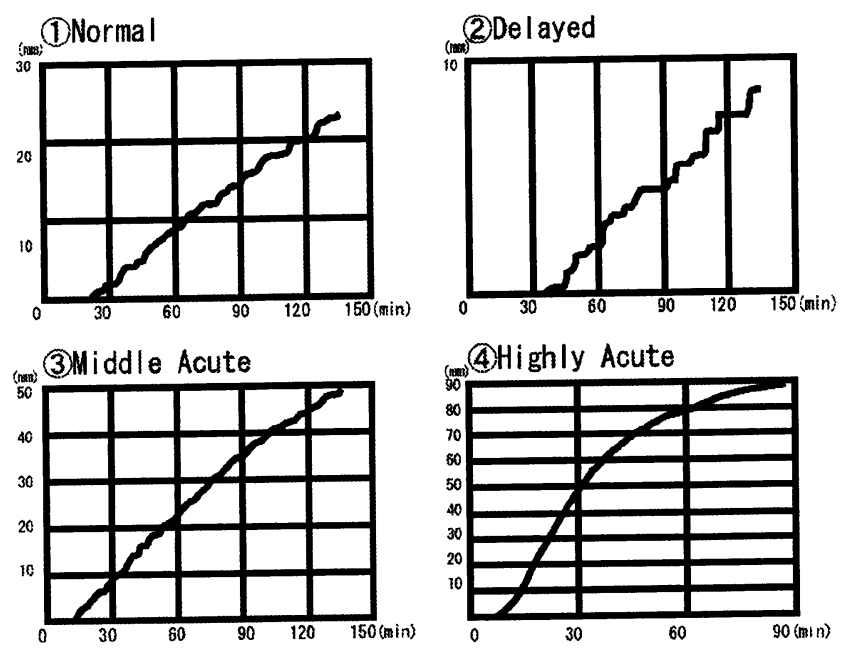

Fig. 1 Sedimentation curve obtained with Quick-Eye.

the holder is set to tilt manually at 60 degrees from the vertical line. The sedimentation distance is read at the meniscus position of the glass tube at $6 \mathrm{~min}$. for the Westergren 1-hour value and at $9 \mathrm{~min}$. for the Westergren 2-hour value after tilting of the tubes. However, reading of the meniscus is very difficult, and the values obtained are somewhat lower than Westergren values. ${ }^{15)}$

Details on the functions of these methods are listed in Table 1.

\section{FACTORS INVOLVING ACCELERATION OF BLOOD SEDIMENTATION RATE}

Although ESR accelerating factors are already well known 
Tabuchi et al.: Current status of ESR determination test.

Table 2 Correlation between ESR and blood components.

\begin{tabular}{|l|r|c|}
\hline Accelerator & Latent time & Sedimentation rate \\
\hline Hemoglobin & 0.70 & -0.57 \\
\hline Albumin & 0.31 & -0.47 \\
\hline alpha-1 globulin & -0.49 & 0.68 \\
\hline alpha-2 globulin & -0.48 & 0.70 \\
\hline beta globulin & -0.33 & 0.46 \\
\hline gamma globulin & -0.49 & 0.54 \\
\hline Total protein & -0.21 & n.s. \\
\hline White cell count & n.s. & 0.31 \\
\hline Rheumatoid factor & -0.23 & 0.44 \\
\hline CRP & -0.41 & 0.74 \\
\hline
\end{tabular}

Latent time and Sedimentation rate were automatically calculated by a build-in computer in Quick-eye $(N=111)$ The number in each box represents correlation coefficient $(=r)$. n.s.; not significant.

Italics show high $r$-value.

from clinical and rheological considerations, all such findings were obtained by obsolete laboratory testing methods. Recent technology enables more accurate and precise test results. However, there is no study on which factors using recent kinds of reliable laboratory data. Thus, we studied which factors using the Quick-Eye sedimentation curves and sedimentation rate values (Table 2). Complete blood count and blood clinical chemistry data were studied for comparison.

The latent time was strongly influenced by hemoglobin content in red cells $(r=0.70)$, followed by alpha 1 fraction $(r=$ -0.49 ; its largest component is antitrypsin, at $300 \mathrm{mg} / \mathrm{d} l)$, alpha 2 fraction ( $r=-0.48$; its largest component is haptoglobin, at $540 \mathrm{mg} / \mathrm{d} l)$, gamma globulin $(r=-0.49$; its concentration is $800-1,800 \mathrm{mg} / \mathrm{d} l$ ), and CRP (a component of the gamma globulin fraction, the concentration of which is less than 1 $\mathrm{mg} / \mathrm{d} l$ in normal condition and increases 10 to 1,000 times in cases of acute inflammation). Although hemoglobin had less effect on the sedimentation rate, sedimentation rate was influenced by CRP, antitrypsin, and haptgrobin. CRP had the strongest effect on acceleration of ESR, while gamma globulin had a less strong effect. Fibrinogen distributing between beta and gamma fractions has been considered a strong accelerator of ESR. In our study, fibrinogen concentration was correlated with 1-hour ESR value with an $r$-value of only 0.47 . The Latent time is affected by hemoglobin content. In other words, hemoglobin content affects rouleaux formation strongly, while the sedimentation rate itself will be affected by combined actions of major acute-phase reactants consisting of alpha 1 fraction, alpha 2 fraction and CRP.

\section{DIFFERENCES BETWEEN ESR AND CRP IN CLINICAL SIGNIFICANCE}

ESR has been believed to reflect acute-phase response. However, it has been used for diagnosing and monitoring tuberculosis, a chronic form of inflammation, for many years in Japan. ${ }^{15)}$ Hypergammaglobulinemia, such as that in liver cirrhosis and rheumatoid arthritis ${ }^{16), 17)}$ demonstrates acceleration



Fig. 2 Mechanism of ESR acceleration.

of ESR, although gamma globulin is not an acute-phase reactant. CRP and fibrinogen increase in plasma when acute inflammation occurs, and can accelerate ESR. Red cell count and hematocrit are accelerators, although not acute phase reactants. ${ }^{18)}$ Thus, ESR accelerators can be divided into 3 groups: those with high content of acute-phase proteins, hyperglobulinemia and anemia.

In our Quick-Eye curve analysis, initial latent time of ESR correlated strongly with hemoglobin, while sedimentation rate correlated strongly with CRP and alpha-1 and alpha- 2 globulin fractions (Table 2). Between Westergren 1-hour value and fibrinogen concentration, an $r$ of $0.5517(n=45)$ was demonstrated in our experiments, which was not high. Thus, the initial latent time of the curve reflects rouleaux formation rate and zeta potential of red cells, and the sedimentation rate reflects interaction between red cell shape and plasma components. ${ }^{19-21)}$

ESR is surely a non-specific and non-diagnostic test, but is very useful in detecting disease conditions and performance status in chronic inflammation. ${ }^{22-26)}$ Compared with ESR, both CRP and white cell count are the best markers for acute inflammations. Mobilization of white cells and release of CRP is due to acute inflammatory response, while acceleration of ESR is an overall outcome of acute and chronic inflammatory changes and anemia (Fig. 2). The correlation between CRP and white cell count was $0.367(n=248)$, but the correlation between CRP and neutrophil count was $0.392(n=248) .{ }^{27)}$ White cell count determination cannot reflect both CRP and ESR. The half-life of CRP is short, but those of gamma globulin and red cells are long. Acceleration of ESR is often observed in noninflammatory conditions. Therefore, we should not compare ESR and CRP in the same table. ${ }^{22)-29)}$

\section{FUTURE OF THE ESR TEST}

ESR is a simple, inexpensive and reliable laboratory test that clinician have used for decision-making for over 70 years. ${ }^{30)-32)}$ Despite this fact, many have criticized the test because of its lack of specificity and inadequacy for laboratory automation. CRP is often used as a substitute for ESR. However, the mech- 
anism of acceleration of ESR is different from that of elevation of CRP. Since ESR is a comprehensive sign of inflammation and anemia, it should be used correctly and appropriately. Currently, various types of rapid ESR test systems are marketed. After confirming the precision and accuracy of the system you choose, such a conventional test system should be used for health check-up and promotion, referring to the ICSH reference method.

\section{REFERENCES}

[1] ICSH. ICSH recommendations for measurement of erythrocyte sedimentation rate. J Clin. Pathol. 1993. 46. 198-203.

[2] ICSH. Reference method for the erythrocyte sedimentation rate (ESR) test on human blood. Br J Haematol. 1973. 24. 671-673.

[3] ICSH. Guidelines on selection of laboratory tests for monitoring the acute phase response. J Clin Pathol. 1988. 41. 12031212.

[4] Stuart MC.: Evaluation of a $200 \mathrm{~mm}$ long vacuum aspiration tube for measurement of erythrocyte sedimentation rate. J Clin Pathol. 1991. 44. 429-430.

[5] ICSH. Protocol for type testing equipment and apparatus used for hematological analysis. J Clin Pathol. 1978. 31. 275-279.

[6] Fahreus R.: The suspension-stability of the blood. Acta Med Scand. 1921. 55. 1-228.

[7] ImafukuY, Yoshida H.: Do we really need the ESR test? JJCLA 2001 Vol.26. No.2. 91-95.

[8] Bull BS., Brailford JD.: The zeta sedimentation ratio. Blood. 1972. 550-559.

[9] Bucher WC., Gall EP., Woodworth R.: Zeta sedimentation ratio in rheumatic disease. Amer J Clin Pathol. 1975. 64.613-617.

[10] Miyazima H., Kojima K., Yamada T., et al.: Clinical evaluation for a rapid ESR apparatus. Clinical Laboratory Instruments and Reagents (KIKI-SHIYAKU). 1999. 22. 483-487 (in Japanese).

[11] Stuart MC.: Assessment of Diesse Ves-matic automated system for measuring erythrocyte sedimentation rate. J Clin Pathol 1991. 44. 946-949.

[12] Plebani M., DeToni S., Sanzari MC.: The test 1 automated system; a new method for the erythrocyte sedimentation rate. Amer J Clin Pathol. 1998. 110. 334-340.

[13] Tabuchi T., Tominaga T., Tatsumi N.: Evaluation for utility of quick ESR device (Quick-eye). JPN J Laboratory Hematology. 2001. 02. (abstract)

[14] Tabuchi T., Tominaga T., Tatsumi N.: Problem relating rapid methods for ESR determination and its solution, South-eastern Asian J Tropical Medicine. (in preparation)

[15] Kanai M.: ESR. In Kanai's Manual of Clinical Laboratory
Medicine. 31st Ed. 1999. pp. 362-365

[16] Furota A.: Blood components involving acceleration of erythrocyte sedimentation rate. Health Evaluation and Promotion (in press).

[17] Kashiwazaki S.: Diagnosis of collagen and rheumatic diseases. In Clinics for Collagen Diseases and Rheumatic Diseases. edited by Kashiwazaki S. Nankodo Co. (Tokyo). 1994. p.15.

[18] Reinhart WH., Singh A., Straub OW.: Red cell aggregation and sedimentation; the role of the cell shape. Br J Haematol. 1989. 71. 551-556.

[19] Fabry TL.: Mechanism of erythrocyte aggregation and sedimentation. Blood. 1987. 70.1572-1576.

[20] Koch HJ.: Posible role of erythrocyte sedimentation rate, hematocrit and oxygen supply of tissue in clinical investigation. Cardiology. 1995. 86. 177-178.

[21] Robins EB., Khan AJ., Atrak T., et al.: Erythrocyte sedimentation rate. Clinical Pediatrics. 1993. Nov. 681-683.

[22] Tinetti ME., Schmidt A., Baum J.: Use of the erythrocyte sedimentation rate in chronically ill, elderly patients with a decline in health status. Am J Med. 1986. 80. 844-848.

[23] Katz PR., Gutman SI., Richman G., et al.: Erythrocyte sedimentation rate and C-reactive protein in the elderly. Clin Chem. 1989. 35/3., 466-468.

[24] Dinant GJ., Habet PA., van der Tempel H., et al.: Discminating ability of plasma viscosity and erythrocyte sedimentation rate; a prospective study at the rheumatology outpatient department. Scand J Rheumatol. 1992. 21. 186-189.

[25] Wolfe F.: Comparative usefulness of C-reactive protein and erythrocyte sedimentation rate in patients with rheumatoid arthritis. J Rheumatol. 1997. 24. ri1477-1485.

[26] Johansson J-E., Sigursson T., Holmberg L., et al.: Erythrocyte sedimentation rate as a tumor marker in human prostatic cancer. Cancer. 1992. 70. 1556-1562.

[27] Tsuda I., Katagami T., Takubo T., et al.: Relationship between CRP and white cell count analyzed in outpatients. Medicine and Biology. 1998. 136. 99-103.

[28] Kinoshita M., Sagawa H., Tsuda I., et al.: Re-evaluation of ESR in general medicine by means of multifactor analysis. Medicine and Biology. 1997. 135. 37-42.

[29] Tatsumi N., Tsuda I., Fukumori T., et al.: Determination of CRP and its clinical significance. Readout. 1999. 19. 55-61.

[30] Brigden M.: The erythrocyte sedimentation rate; still a helpful test when used judiciously. Postgraduate Medicine. 1998. 103. 257-274.

[31] Brigden ML.: Clinical utility of the erythrocyte sedimentation rate. Am Fam Physician. 1999. 60. 1443-1450.

[32] Bedel SE., Bush BT.: Erythrocyte sedimentation rate; from folklore to facts. Am J Med. 1985. 78. 1001-1009. 